\title{
Cholesterol granuloma of the breast suspected as breast carcinoma
}

\author{
Takaaki Fujii, Reina Yajima, Hiroki Morita, Satoru Yamaguchi, Soichi \\ Tsutsumi, Takayuki Asao, Hiroyuki Kuwano
}

\begin{abstract}
Introduction: Cholesterol granuloma of the breast is a rare benign condition which is difficult to differentiate from breast cancer. Case Report: We herein report the case of a 56-yearold Japanese asymptomatic female who, on a computed tomography examination for sigmoid colon cancer, was found to have a palpable nodule in the upper external quadrant of the left breast. Breast carcinoma could not be ruled out on the basis of clinical and radiological findings, but excisional biopsy revealed cholesterol granuloma. A histological examination implied that this case of cholesterol granuloma might have been related with mammary duct ectasia. Conclusion: Although cholesterol granuloma is a rare disorder in the breast, this case indicated that a better awareness of this benign breast disease is important for avoiding misdiagnosis and unnecessary surgery.
\end{abstract}

Keywords: Cholesterol granuloma, Breast carcinoma, Ductal ectasia

$* * * * * * * * *$

Takaaki Fujii' ${ }^{1}$, Reina Yajima $^{1}$, Hiroki Morita ${ }^{1}$, Satoru Yamaguchi $^{1}$, Soichi Tsutsumi ${ }^{1}$, Takayuki Asao ${ }^{1}$, Hiroyuki Kuwano ${ }^{1}$

Affiliations: 1 Department of General Surgical Science, Graduate School of Medicine, Gunma University, Gunma, Japan.

Corresponding Author: Takaaki Fujii, MD, PhD, Department of General Surgical Science, Graduate School of Medicine, Gunma University, 3-39-22 Showa-machi, Maebashi, Gunma 371-8511, JAPAN; Ph: +81-027-220-8224; Fax: +81-027-220-8230; Email: ftakaaki@med.gunma-u.ac.jp

Received: 20 June 2013

Accepted: 27 July 2013

Published: 01 December 2013
Fujii T, Yajima R, Morita H, Yamaguchi S, Tsutsumi S, Asao T, Kuwano H. Cholesterol granuloma of the breast suspected as breast carcinoma. International Journal of Case Reports and Images 2013;4(12):723-726.

$* * * * * * * * *$

doi:10.5348/ijcri-2013-12-420-CR-14

\section{INTRODUCTION}

Cholesterol granuloma consists of fibrous granulation tissue containing cholesterol crystals with foreign body giant cells. Cholesterol granuloma occurs commonly in the mastoid region and in various organs such as the peritoneum, kidney, parotid gland, thyroglossal duct, lymph node, liver, and spleen. However, cholesterol granuloma of the breast is a very rare tumor-like lesion and has been rarely reported. We herein report a rare case of cholesterol granuloma of the breast. Cholesterol granuloma should be considered in the differential diagnosis of breast lesion suspected to be malignant [1-6].

\section{CASE REPORT}

A 56-year-old Japanese asymptomatic female was admitted to our hospital with a diagnosis of sigmoid colon cancer. Computed tomography (CT) scan of her chest and abdomen was done as a preoperative examination of her colon cancer, and showed a nodule in the left breast (Figure 1). The patient had no family history of breast cancer, no history of mammary trauma or biopsy and no history of lipid metabolism abnormality.

On admission, physical examination revealed a $2.0 \times 2.0 \mathrm{~cm}$ palpable nodule which was elastically firm and mobile in the upper external quadrant of the left breast with no associated skin findings. There was no axillary or supraclavicular lymphadenopathy, and no 
abnormal nipple discharge. Mammography showed a $2.0 \mathrm{~cm}$ in diameter high-density shadow lesion, which corresponded to the palpable mass, with partly ill-defined margins and without specula or microcalcification (Figure 2). An ultrasonography of the left breast revealed a hypoechoic and relatively irregularly shaped mass with an ill-defined margin (Figure 3). A core needle biopsy of the lesion showed fibrosis and a small granulomatous lesion infiltrated by lymphocytes and foamy histiocytes, and there was no evidence of malignancy. From the physical examination, CT scan, ultrasonographic findings, a diagnosis of breast cancer could not be ruled out, and an excisional biopsy was also done when the sigmoidectomy was performed.

The surgical specimen measured $2.4 \mathrm{~cm}$ in diameter and was a cystic and firm mass. The histological evaluation revealed a cystic mass surrounded by fibrous granulation tissue in which needle-like crystals were deposited and accompanied by giant cells (Figure 4). These findings were compatible with cholesterol granuloma. Our follow-up, the patient was remained uneventful since 60 months.

\section{DISCUSSION}

We herein report a rare case of cholesterol granuloma of the breast. Cholesterol granuloma of the breast is composed of fibrous granulation tissue in which cholesterol crystals accompanied by giant cells are deposited. The pathogenesis of cholesterol granuloma is not fully understood. Physical and infectious factors have been considered and some reports postulate that they are caused by a reactive response to an unknown stimulus. In the breast, cholesterol granuloma is thought to represent a rare manifestation of duct ectasia. Lipid-rich material is normally found in ectatic ducts. It is postulated that leakage of cholesterol crystals from the ectatic ducts causes a foreign body giant cell reaction which results in granuloma formation. Mammary duct ectasia is a disorder of middle-aged women. Mammary duct ectasia is a frequent disorder of the breast that has a wide variety of clinical manifestations, and this disorder sometimes involves an extensive disruption of the dilated duct and is occasionally noticed as a palpable mass. In rare instances the mass, microscopically, contains cholesterol crystals. In the pathologic specimen in this case, a cystic lesion surrounded by fibrous granulation tissue, chronic inflammation, cholesterol crystals, and scattered multinucleated giant cells was observed. The lesion was considered to be cholesterol granuloma associated with duct ectasia [1-6].

The main concern with breast cholesterol granuloma is performing differential diagnosis. Clinically, it resembles breast cancer. The clinical and radiological features of cholesterol granuloma mimic those of a carcinoma of the breast. Thus, it is difficult to distinguish cholesterol granuloma from carcinoma of the breast based on the

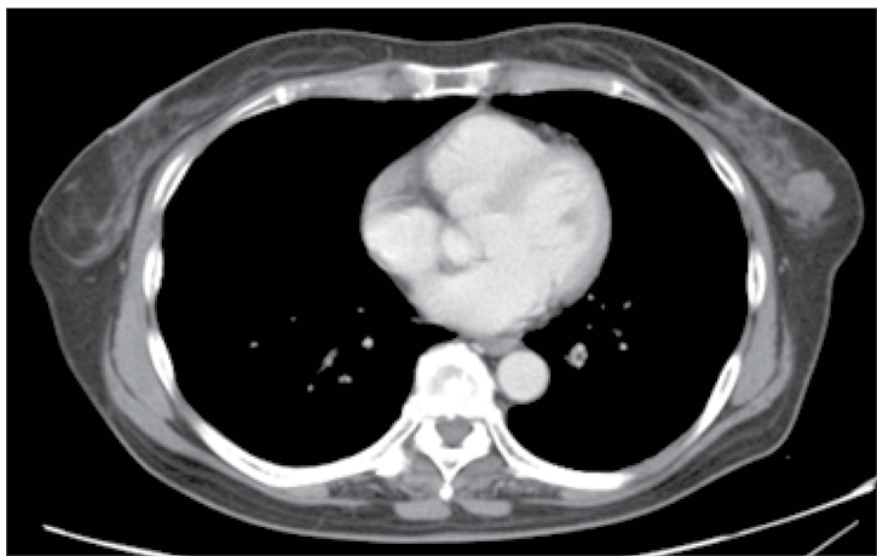

Figure 1: Computed tomography scan of chest showing a nodule in the left breast.

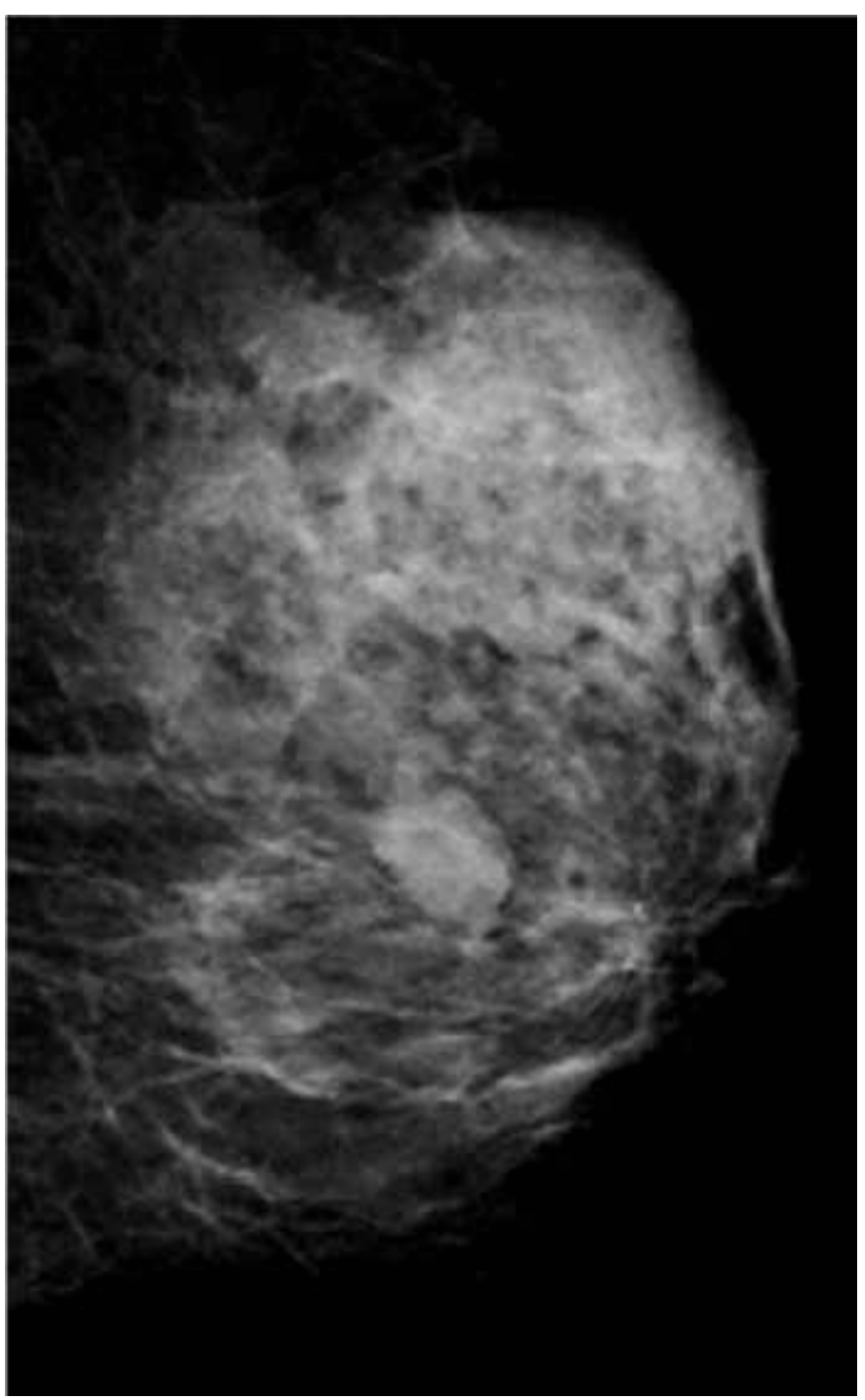

Figure 2: Mammography showing a $2.0 \mathrm{~cm}$ in diameter highdensity shadow lesion, which corresponded to the palpable mass, with partly ill-defined margins and without specula or microcalcification. 


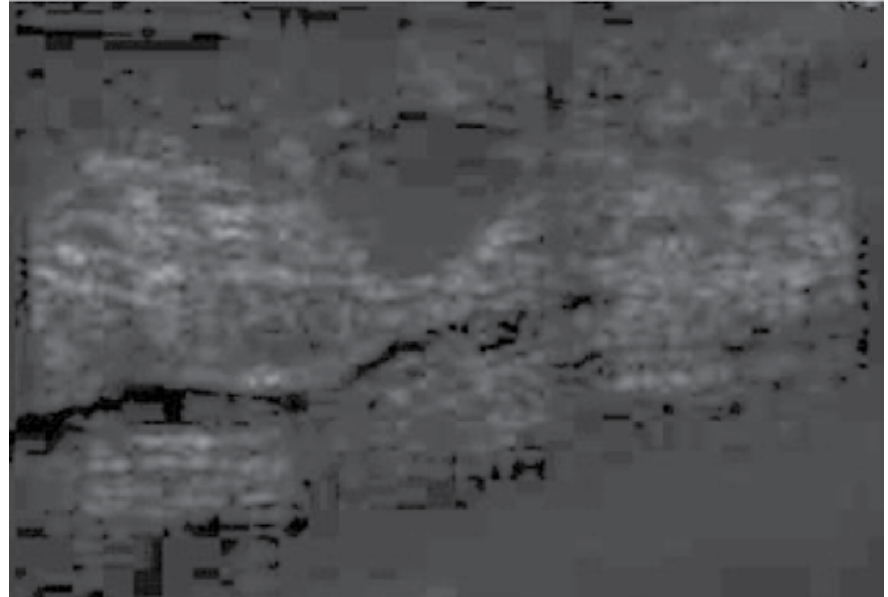

Figure 3: An ultrasonography of the left breast revealed a hypoechoic and relatively irregularly shaped mass with an illdefined margin.
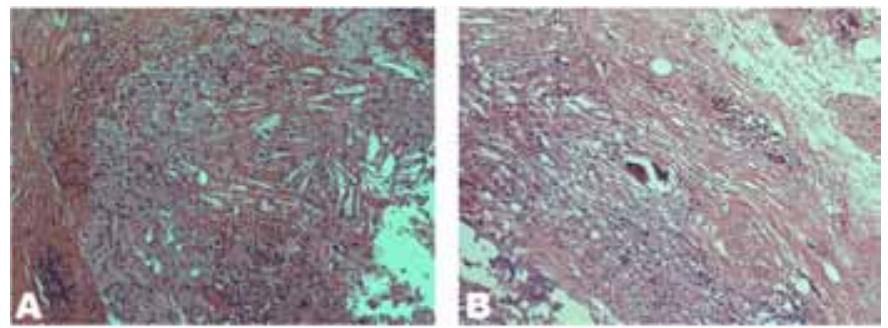

Figure 4: The histological evaluation revealed a cystic mass surrounded by fibrous granulation tissue in which (A) needlelike crystals were deposited, (B) accompanied by giant cells (H\&E stain, 200x).

imaging results. The diagnosis of cholesterol granuloma is histologic. It is not difficult to distinguish between cholesterol granuloma and breast cancer histologically, and core needle biopsy is thought to be useful for differential diagnosis. However, it should be noted that breast cholesterol granuloma could be accompanied by cancer. Thus, cases in which malignancy cannot be ruled out clinically, a complete removal of the tumor helps in making a definitive diagnosis [1-6].

\section{CONCLUSION}

We here reported a rare case of breast cholesterol granuloma mimicking breast cancer. Reports on breast granuloma are rare. However, duct ectasia itself, which is related with cholesterol granuloma, is a very frequent disorder. To make an accurate diagnosis of this rare disorder and rule out the possibility of cancer, a histological examination is required.

\section{Author Contributions}

Takaaki Fujii - Substantial contributions to conception and design, Acquisition of data, Drafting the article, Revising it critically for important intellectual content, Final approval of the version to be published

Reina Yajima - Substantial contributions to conception and design, Acquisition of data, Drafting the article, Revising it critically for important intellectual content, Final approval of the version to be published

Hiroki Morita - Substantial contributions to conception and design, Drafting the article, Final approval of the version to be published

Satoru Yamaguchi - Substantial contributions to conception and design, Drafting the article, Final approval of the version to be published

Soichi Tsutsumi - Substantial contributions to conception and design, Drafting the article, Final approval of the version to be published

Takayuki Asao - Substantial contributions to conception and design, Drafting the article, Final approval of the version to be published

Hiroyuki Kuwano - Substantial contributions to conception and design, Drafting the article, Revising it critically for important intellectual content, Final approval of the version to be published

\section{Guarantor}

The corresponding author is the guarantor of submission.

\section{Conflict of Interest}

Authors declare no conflict of interest.

\section{Copyright}

(C) Takaaki Fujii et al. 2013; This article is distributed under the terms of Creative Commons attribution 3.0 License which permits unrestricted use, distribution and reproduction in any means provided the original authors and original publisher are properly credited. (Please see www.ijcasereportsandimages.com/copyright-policy.php for more information.)

\section{REFERENCES}

1. Reynolds HE, Cramer HM. Cholesterol granuloma of the breast: a mimic of carcinoma. Radiology 1994;191(1):249-50.

2. Osada T, Kitayama J, Nagawa H. Cholesterol granuloma of the breast mimicking carcinoma: report of a case. Surg Today 2002;32(11):981-4.

3. Garofalo S, Casolino C, Accurso A, Falleti J. Choesterol granuloma of the breast with unusual ossification features (osseous metaplasia). Pathol Res Pract 2008;204(5):353-6.

4. Smith GL, Hicks P, Wijesinghe DP, Holme TC. Cholesterol granuloma of the breast presenting as an intracystic papilloma. Br J Radiol 1997;70(839):11789. 
5. Ishizaki M, Ohsumi S, Takashima S, Mandai K. Two cases of cholesterol granuloma of the breast. Breast Cancer 2001;8(2):158-61.
6. Furuhira C, Ohshima A, Shimada K, et al. A case of breast cholesterol granuloma accompanied by cancer. Breast Cancer 2004;11(2):210-3.
Access full text article on other devices

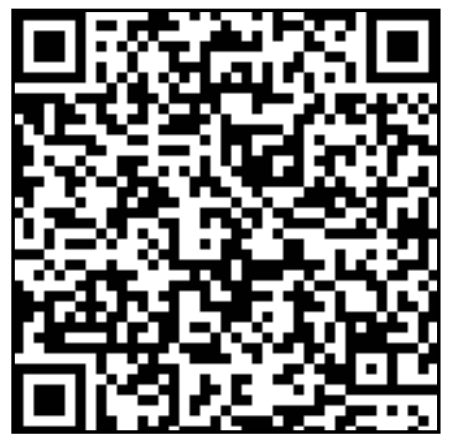

Access PDF of article on other devices

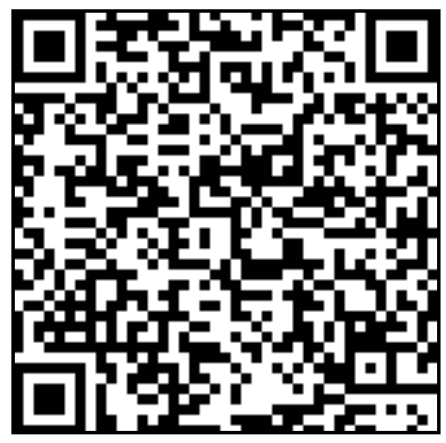

